\title{
Primary Malignant Parotid Melanoma: Searching for a Rare Entity
}

\author{
Ithzel Maria Villarreal1, Beatriz Arellano1, Jeanette Sáenz1, Luz Martin², \\ José Ramón Castello ${ }^{3}$, José Ramón García-Berrocal ${ }^{1 *}$ \\ ${ }^{1}$ Department of ENT, "Puerta de Hierro-Majadahonda" University Hospital, Madrid, Spain \\ ${ }^{2}$ Department of Pathology, "Puerta de Hierro-Majadahonda" University Hospital, Madrid, Spain \\ ${ }^{3}$ Department of Plastic Surgery, "Puerta de Hierro-Majadahonda" University Hospital, Madrid, Spain \\ Email: *irgarciab@yahoo.com
}

Received 7 July 2014; revised 5 August 2014; accepted 1 September 2014

Copyright (C) 2014 by authors and Scientific Research Publishing Inc.

This work is licensed under the Creative Commons Attribution International License (CC BY).

http://creativecommons.org/licenses/by/4.0/

(c) (i) Open Access

\begin{abstract}
Objectives: The aim of this paper is to establish a correct approach to the diagnosis of a primary malignant melanoma of the parotid gland. Methods: We present a case of a patient with a painless mass situated in the left parotid region after an initial cytological and radiological study suggesting a Warthin tumor whose treatment involved a total parotidectomy with a facial nerve reconstruction. Results: The histopathological result showed a malignant melanoma that after various studies and evaluations showed no other primary origin different from the parotid gland itself. Conclusions: Salivary gland melanomas are relatively rare entities that when being of a primary origin and localized in the parotid gland become even more uncommon. Upon a melanoma found in the parotid gland, the first suspicion should be a metastatic origin, being the most common sites the oral cavity and the skin from the head and neck region.
\end{abstract}

\section{Keywords}

Primary Parotid Melanoma, PET-Scan, S100 Protein, Facial Nerve Reconstruction

\section{Introduction}

Malignant melanoma is one of the most aggressive malignant tumors accounting for $15 \%$ of all cancers [1] and represents approximately $0.7 \%$ of all malignant neoplasms within the parotid gland [2]. They are characterized by a difficult late diagnosis and poor prognosis. Most of them are considered to be metastasis although sometimes the primary origin cannot be established [1]. Primary melanoma of the parotid gland is considered extremely rare [2] [3].

\footnotetext{
*Corresponding author.

How to cite this paper: Villarreal, I.M., Arellano, B., Sáenz, J., Martin, L., Castello, J.R. and García-Berrocal, J.R. (2014) Primary Malignant Parotid Melanoma: Searching for a Rare Entity. International Journal of Otolaryngology and Head \& Neck Surgery, 3, 228-232. http://dx.doi.org/10.4236/ijohns.2014.35042
} 


\section{Case Report}

A 50-year-old male presented at our institution with a 3 months history of a painless mass in the left mandibular angle which had rapidly increased in size. No other symptoms were present and his past medical history was unremarkable.

Physical examination showed a round, painless approximately $1 \mathrm{~cm}$ in size mass located in the left parotid compartment. No other clinical features were perceived.

Complementary studies were done, beginning with an ultrasound that revealed both parotid glands enlarged finding also in the left side a solid mass within the superficial lobe. It was well defined, slightly lobulated, approximately $3.8 \times 2 \mathrm{~cm}$ large, hypoechogenic and heterogeneous with very little vascularization, with contrast dye the mass enhanced except for small filiform vessels found interiorly and a small enhancing found peripherically that suggested a Warthin tumor as a first possible diagnosis. Ultrasonography guided fine needle aspiration cytology was informed as inconclusive. Consequently an MRI (Magnetic Resonance Imaging) was also performed, in which a small intraparotid nodule with heterogeneous signal also suggesting a possible Warthin tumor as well as the ultrasound was described but since it did not have the typical enhancement pattern of a Warthin tumor, a pleomorphic adenoma had to be ruled out too (Figure 1). A PET-scan performed before treatment showed no other sites affected.

With a suspicion of a possible pleomorphic adenoma a left superficial lobe parotidectomy was scheduled. During the intervention, we observed some dark areas in the tumor making necessary an intraoperative biopsy that confirmed a melanoma (T3N0M0). The intervention had to be transformed into a total parotidectomy and facial nerve reconstruction with a sural nerve graft (Figure 2).

Other complementary studies were done searching for a possible primary tumor, considering that a primary malignant melanoma of the salivary glands is so uncommon.

Digestive tests such as colonoscopy and an upper endoscopy reported the following results: in the colonoscopy the caecum, the ileocaecal valve and the appendicular orifice were identified and from there down to the rectum, 2 small polyps in the hepatic angle and rectum were the only pathological findings which were histologically analyzed being identified as hyperplastic polyps, and the upper endoscopy showed a small hiatal hernia and a Schatzki ring with no other pathological findings

A cranial/brain MRI was performed and informed as normal and a thoracic-abdominal Computerized Tomography (CT scan) showed no signs of a possible primary tumor in this area.

He also was evaluated by a Dermatologist, Neurologist and Ophthalmologist not finding any suggestion in the physical examination or imaging testing that lead to a suspicion of a possible primary tumor in their areas of expertise. The histopathological final result confirmed an intraglandular melanoma whose cells were S100 positive, melan A positive and HMB-45 positive. No lymphoid tissue was identified on the periphery of the tumor (Figure 3). He was referred then to the department of Oncology for evaluation whose decision was to make a close follow-up. A 6 months follow-up has shown no surgical complications. Diagnostic imaging performed recently (PET-scan) showed postsurgical changes and was free of highly metabolic activity in the parotid region and in other areas that could suggest extraglandular primary tumor localization.

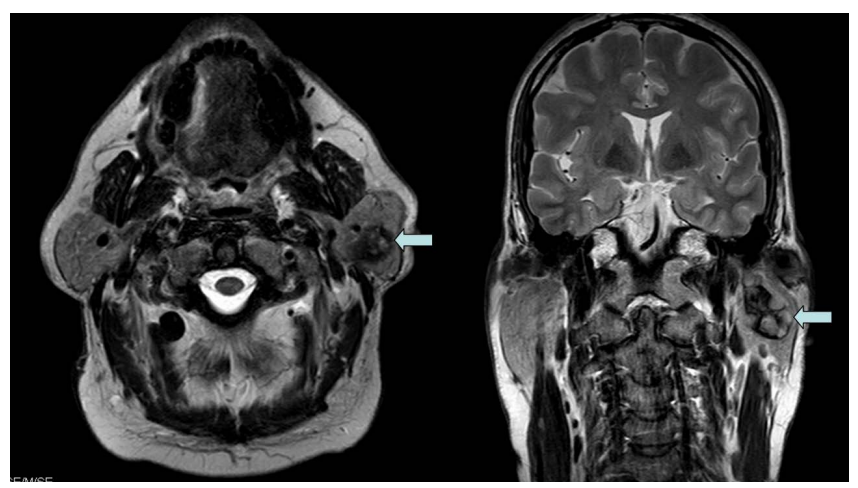

(a)

(b)

Figure 1. Axial and coronal T1-weighted (a) and T2 (b) respectively MRI, demonstrates a high-signal-intensity in T1, heterogeneous mass in the left parotid gland (arrow). 


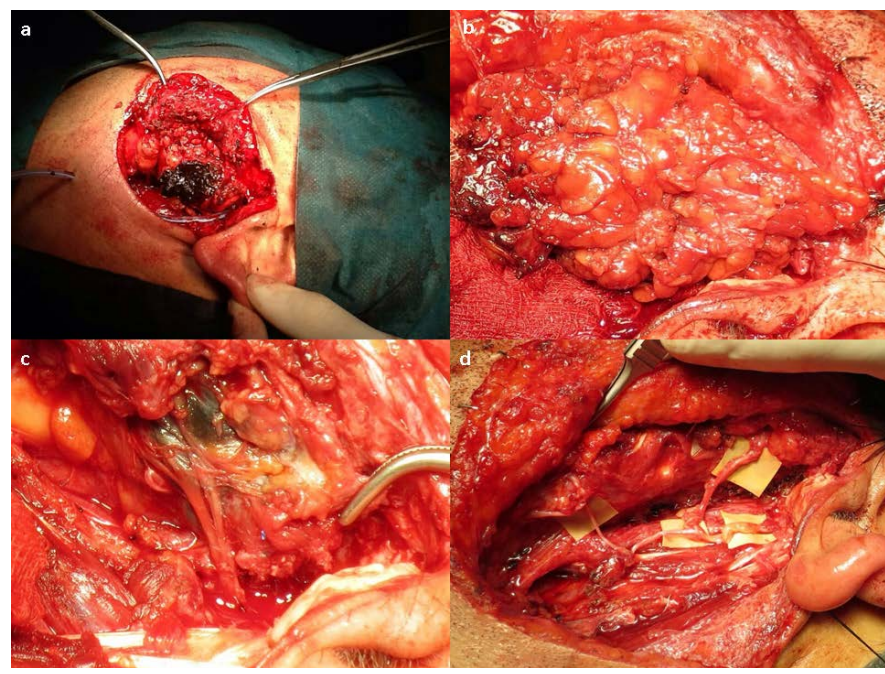

Figure 2. Macroscopic vision of a pigmented area of firm consistency, $2.2 \times 2 \times 1.8 \mathrm{~cm}$ in size occupying the postero-inferior border of a $6.5 \times$ $4.5 \times 3 \mathrm{~cm}$ parotid gland. Borders had poor differentiation (a) (b). The facial nerve is found to be penetrating the tumor and infiltrated by the mass (c). A facial nerve was reconstructed by grafting of the cutaneous branch of the femoral nerve (d).

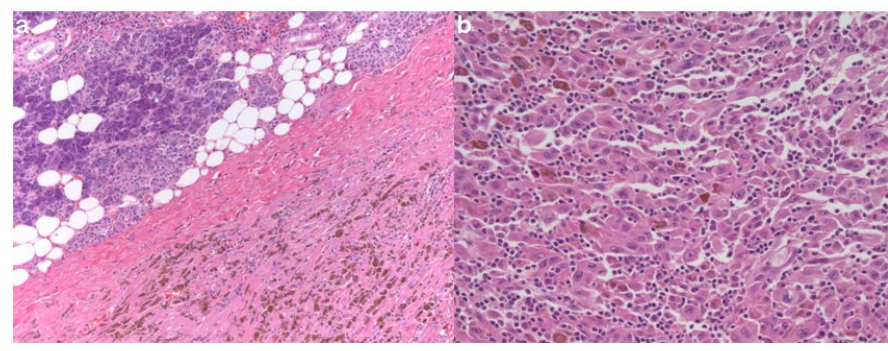

Figure 3. The tumor is separated from the adjacent parotid tissue, without evidence of lymph node (Hematoxylin/eosin $\times 100$ ) (a). The tumor consists of epitheliod neoplastic cells showing prominent nucleoli, atypical mitotic figures and areas with melanin pigmentation (Hematoxylin/eosin $\times 400)$ (b).

\section{Review and Discussion}

The diagnosis of a primary malignant melanoma (PMM) of a salivary gland origin has always been controversial. A primary site has to be ruled out always before the confirmation of this diagnosis. Intraorally the hard palate is the most common site (80\%) followed by the entire skin, nose, pharynx, mouth, oesophagus, anogenital region and meninges [4].

Greene and Bernier suggest two basic criteria to rule out PMM [5].

1) Histopathologic demonstration of PMM in the gland.

2) Inability to demonstrate any other primary tumor origin.

Clinical behaviour is unpredictable and variable. Some clinical features of a parotid malignancy at presentation may include a parotid mass or lump, fixity to skin and deep neck structures, pain/painless mass, lymphadenopathy, rapid growth, facial nerve palsy and also tumor fungation [6].

Hypotheses have been made to justify a primary origin in the parotid gland. Greene and Bernier believe they arise from the melanoblasts present in the tissue because the embryonic origin of the parotid gland lies in the oral epithelium where melanoblasts makeup its normal constituents [5]. Takeda et al. found melanocytes or dopa-positive cells (stained using L dopa or 3,4-dihydroxy-L-phenylalanine) in the interlobular duct of the parotid gland during autopsy [3] [5] [6] [8]. Histologically, they are characterized by being a multinodular lobu- 
lated tumor, with cells that show large nuclei, prominent nucleoli and plentiful eosinophilic cytoplasm. Within the tumor cells, brown granular pigment, melanosomes and compound melanosomes are found [1].

The presence of neuromelanin within a tumor or characteristic cytologic features such as intranuclear invagination of the eosinophilic cytoplasm or prominent nucleolation can be helpful diagnostic clues when differentiating melanomas from carcinoma [7].

Immunohistochemistry in these neoplasms is characterized by S100 protein, melan A and HMB-45 positivity [1] [6] [8].

Woodward et al. proposed that four conditions should be met before making a diagnosis of primary parotid gland melanoma [3] [4] [7] [8]:

- The predominant tumor mass or bulk should be intraparotid.

- There should be no identifiable lymph node tissue present in the mass.

- There should be no evidence of malignant melanoma elsewhere after diligent search of eyes, skin, nose, pharynx, mouth, oesophagus, anogenital region or meninges.

- There should be no evidence of previous excision of a malignant melanoma or progressing pigmented lesion. In our case, all of these conditions were fulfilled.

A thorough study should include a careful analysis of the integument, ocular examination, ultrasonography/ CT scan of the abdomen, panendoscopy, PET-scan, and CT scan of thorax and brain [9]. Lopez-Cedrun et al. suggest that even with normal results there is a doubt about the primary origin suggesting the location of the primary malignant melanoma in a hardly explorable internal organ [2].

According to a study by Shah et al., patients submitted to neck dissection for a primitive melanoma of the face, ear or anterior region of the scalp should be considered for parotidectomy [2] [10].

Lymph node involvement seems to be one of the more useful predictive parameters [4]. Metastases involving the intraparotid lymph nodes tend to have well-demarcated interfaces between the capsule of the node and the adjacent salivary gland tissue, in contrast to supposed primary melanoma that tend to infiltrate with poor demarcation [3].

Neck dissection should always be performed because of the high incidence of hidden metastases in these nodal groups [2] [3] [10]. The presurgical PET scan was negative for a possible primary origin which made us decide not to perform a cervical lymphadenectomy.

Radiologically, MRI is shown to be a better option than CT scan because it provides more detailed anatomical information and may better define the position of the parotid mass in relation to the facial nerve [6].

Standard treatment in parotid gland cancers is surgical and a total parotidectomy should be performed [2]. Radiotherapy and chemotherapy are considered to be of low value [3] [11]. In this type of tumors, adjuvant radiotherapy is not part of a regular treatment protocol but it is recommended for high risk patients, based on grade, stage and margin of excision to improve local control.

High incidence of local recurrences is a fact even after apparently adequate surgery also in early and localized lesions. Facial nerve palsy is the most common complication of the surgical management of the parotid malignancies. In our case the facial nerve infiltration leads to its sacrifice and reconstruction.

\section{Conclusion}

Parotid cancer accounts for a histologically and biologically diverse group of neoplasms. Malignant melanoma represents approximately $0.7 \%$ of all malignant neoplasms within the parotid gland [2] [3] [9]. The biological behaviour of primary parotid melanomas is variable and unexpected. Prognosis is influenced by several factors, particularly stage and tumor grade. The diagnostic protocol suggests that a histological diagnosis of a melanoma should always be followed by a thorough search for primary or metastatic disease elsewhere because a primary lesion is a candidate for a proper surgical treatment whereas a metastatic lesion is not [4]. The average survival rate hardly exceeds 2 years [2].

\section{References}

[1] Huguet, P., Gabaldon, M., Sansano, I., et al. (2010) Melanoma maligno amelanótico en un ganglio de glándula parótida. Estudio clinicopatólogico, inmunohistoquímico y molecular de un caso. Revista Española de Patología, 43, 144-147.

[2] Barbieri, M., Gentile, R., Cordone, M.P., et al. (2002) Primitive Malignant Melanoma of the Parotid Gland. ORL, 64, 
297-299. http://dx.doi.org/10.1159/000064131

[3] Tsutsumida, A., Yamamoto, Y., Sekido, M., et al. (2008) Suspected Case of Primary Malignant Melanoma of the Parotid Gland. Scandinavian Journal of Plastic and Reconstructive Surgery and Hand Surgery, 42, 105-107. http://dx.doi.org/10.1080/02844310601004707

[4] Woodwards, R.T., Shepherd, N.A. and Hensher, R. (1993) Malignant Melanoma of the Parotid Gland: A Case Report and Literature Review. British Journal of Oral and Maxillofacial Surgery, 31, 313-315. http://dx.doi.org/10.1016/0266-4356(93)90068-8

[5] Bahar, M., Anavi, Y., Abraham, A., et al. (1990) Primary Malignant Melanoma in the Parotid Gland. Oral Surgery, Oral Medicine, Oral Pathology, Oral Radiology, 70, 627-630. http://dx.doi.org/10.1016/0030-4220(90)90412-L

[6] Malata, C.M., Camilleri, I.G., McLean, N.R., et al. (1997) Malignant Tumours of the Parotid Gland: A 12-Year Review. British Journal of Plastic Surgery, 50, 600-608. http://dx.doi.org/10.1016/S0007-1226(97)90505-1

[7] Prayson, R.A. and Sebek, B.A. (2000) Parotid Gland Malignant Melanomas. Archives of Pathology Laboratory Medicine, 124, 1780-1784.

[8] Gao, N., Li, L.J., Li, Y., et al. (2008) Primary Amelanotic Malignant Melanoma of the Parotid Gland: A Case Report. Journal of International Medical Research, 36, 1435-1439. http://dx.doi.org/10.1177/147323000803600633

[9] Bussi, M., Cardarelli, L., Riontino, E., et al. (1999) Primary Malignant Melanoma Arising in the Parotid Gland: Case Report and Literature Review. Tumori, 85, 523-525.

[10] Renaut, A.J. (1996) Melanoma Arising within the Parotid Salivary Gland a Case Report and Review of Management. European Journal of Surgical Oncology, 22, 201-202. http://dx.doi.org/10.1016/S0748-7983(96)90983-X

[11] Bron, L.P., Traynor, S.J., McNeil, E.B., et al. (2003) Primary and Metastatic Cancer of the Parotid: Comparison of Clinical Behavior in 232 Cases. Laryngoscope, 113, 1070-1075. http://dx.doi.org/10.1097/00005537-200306000-00029 
Scientific Research Publishing (SCIRP) is one of the largest Open Access journal publishers. It is currently publishing more than 200 open access, online, peer-reviewed journals covering a wide range of academic disciplines. SCIRP serves the worldwide academic communities and contributes to the progress and application of science with its publication.

Other selected journals from SCIRP are listed as below. Submit your manuscript to us via either submit@scirp.org or Online Submission Portal.
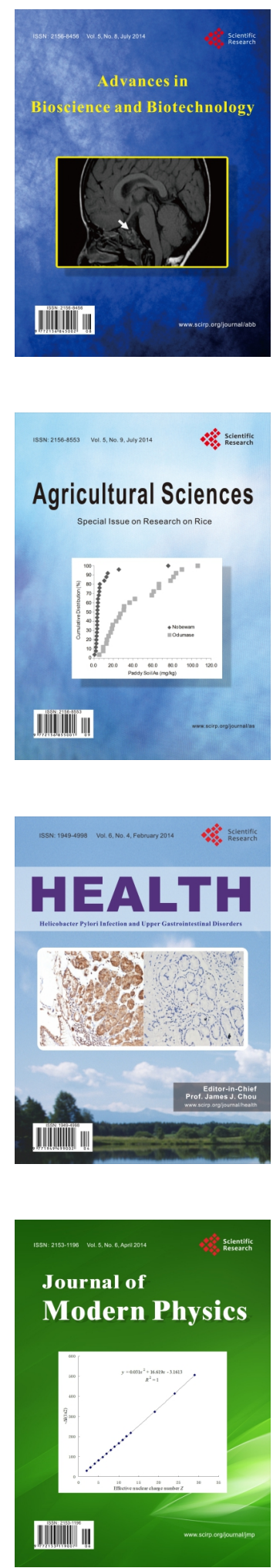
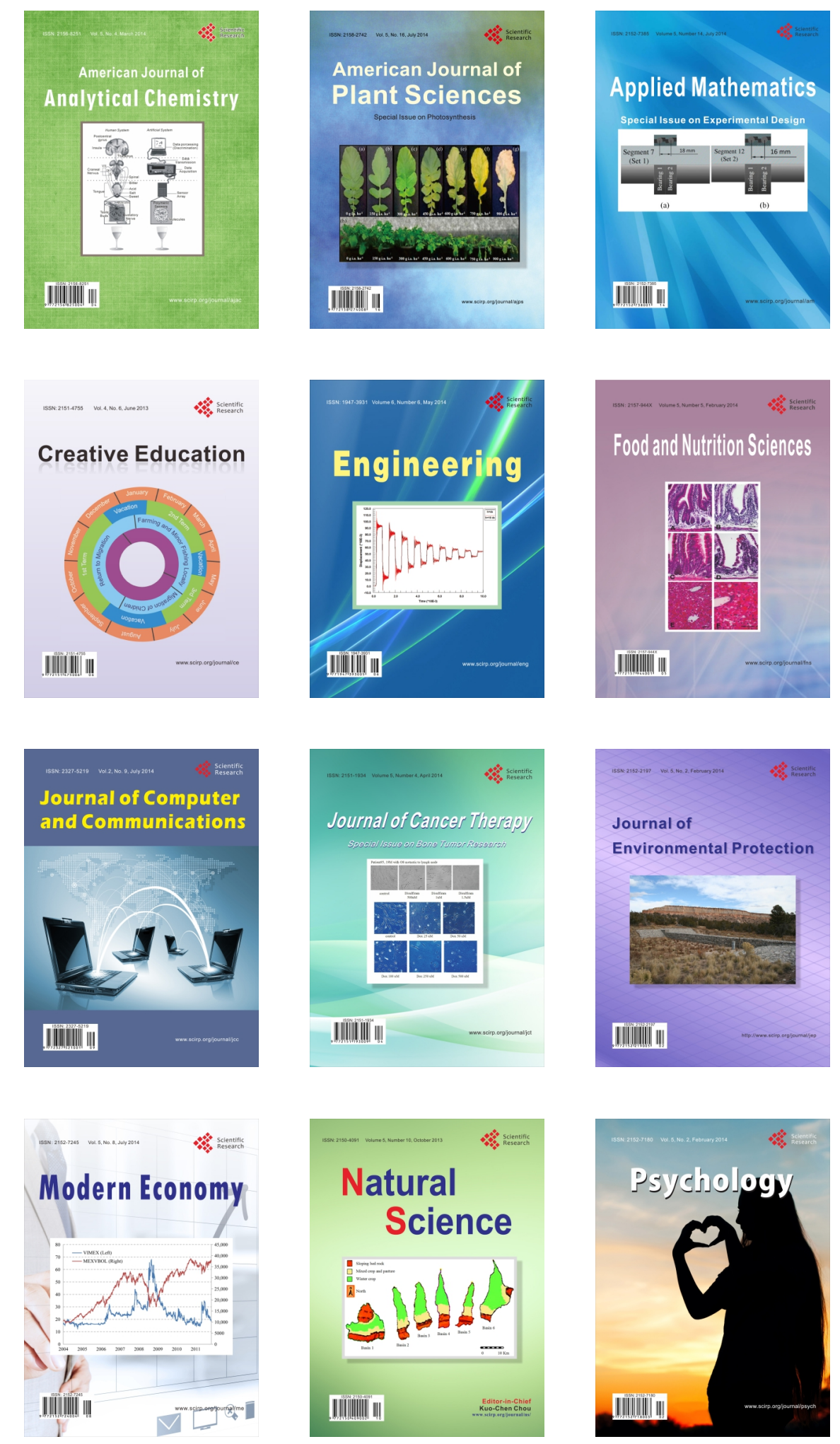